\title{
Clinical and physical signs of water-loss dehydration in older people: a diagnostic accuracy systematic review
}

\author{
L. Hooper ${ }^{1}$, N. J. Attreed ${ }^{1}$, A. M. Channell ${ }^{1}$, D. J. Gilbert ${ }^{1}$, A. C. Heathcote ${ }^{1}$, R. A. Needham ${ }^{2}$, \\ S. C. Ranson ${ }^{1}$, A. C. Smith ${ }^{1}$, S. Ward ${ }^{1}$ \\ ${ }^{1}$ Norwich Medical School, University of East Anglia, Norwich NR4 7TJ, UK and ${ }^{2}$ Gateshead NHS Trust, Gateshead \\ NE9 6SX, UK
}

Water-loss dehydration is common in older people, and associated with increased risk of mortality and disability as well as a wide range of other health problems ${ }^{(1)}$. While biochemical assessment of serum osmolality is the best state (one time) indicator of dehydration in a clinical setting ${ }^{(2)}$ it is not available day to day in the community, primary or residential care. A valid, simple and non-invasive screening test for dehydration for older adults in the community is needed.

We assessed the diagnostic accuracy of minimally invasive clinical and physical signs that may be useful to screen for impending and/ or current water-loss dehydration in older people by systematically reviewing studies in people aged 65 years or more ${ }^{(3)}$.

We searched MEDLINE, EMBASE, CINAHL and LILACS to November 2011 for studies where a reference standard (serum osmolality or weight change) and at least one index test (such as dry axilla, mucous membranes, furrowed tongue, slow capillary refill time, poor skin turgor, orthostatic hypotension, dark urine, raised urine specific gravity, low urine volume, sunken eyes, rapid pulse, bioelectrical impedance, postural pulse increment or postural dizziness) were both assessed in people aged $65+$. Where such data had been collected we contacted authors to ask for access to their data to allow us to calculate $2 \times 2$ tables. 9688 titles and abstracts were assessed, 398 full text papers collected, emails or letters written to 82 study authors and 19 datasets included in the systematic review. Data sets were data extracted independently in duplicate and assessed for validity using the QUADAS tool ${ }^{(4)}$.

Study validity was variable. For example, while some studies measured the reference standard and index test within minutes of each other, some left gaps of several days, which may be problematic as hydration status can change over hours.

There were rarely at least 4 studies assessing a single clinical sign, so meta-analysis was not useful and exploration of heterogeneity unhelpful. Only reporting fatigue and reporting missing some drinks between meals were possible individual markers of early water-loss dehydration (with sensitivity $>0.60$ and specificity $>0.75$ ) but these were assessed in small samples in single studies. Other signs with some diagnostic utility (those with positive likelihood ratios, LR,$+>2$ or negative likelihood ratios, LR-, $<0.5$ in at least 2 studies, or in one study with $>100$ participants) included:

- Fluid intake (from food and drink combined, $<1.7 \mathrm{~L} / \mathrm{d}$ in men, $<1.3 \mathrm{~L} / \mathrm{d}$ in women)

- $\quad$ Body temperature $\left(>38.3^{\circ} \mathrm{C}\right)$

- Dry inside cheek (dry vs. damp)

- Dry axilla (underarm, dry vs. damp)

- Mini-mental state exam (Score $<10$ of 30, MMSE is an assessment of cognition)

We found insufficient evidence to pinpoint any single sign sensitive and specific enough to use on its own as a marker of early dehydration, although further primary research on whether older people report fatigue or report missing drinks between meals is warranted. Research is needed to assess whether signs may be used together to form a simple tool to detect or rule out early water-loss dehydration in the community.

1. Stookey JD, Purser JL, Pieper CF, Cohen HJ (2004) J Am Geriatr Soc 52, 1313-20.

2. Thomas DR, Cote TR, Lawhorne L, Levenson SA, Rubenstein LZ, Smith DA et al. (2008) J Am Med Dir Assoc 9, $292-301$.

3. Hooper L, Culp KR, Stookey JD, Ritz P, Campbell WW, Mack GW et al. (2011) The Cochrane Library;Protocol (Issue 7):CD009647-DOI:10.1002/ 14651858

4. Reitsma JB, Rutjes AWS, Whiting P, Vlassov VV, Leeflang MMG, Deeks JJ (2009) Ch 9. In: Deeks JJ, Bossuyt PM, Gatsonis C, editor(s). Cochrane Handbook for Systematic Reviews of Diagnostic Test Accuracy Version 1.0.0. 